\title{
Image-Based Gender Prediction Model Using Multilayer Feed-Forward Neural Networks
}

\author{
${ }^{1}$ Mohamed Yousif Elmahi, ${ }^{2}$ Elrasheed Ismail Mohommoud Zayid \\ ${ }^{1}$ Department of Computer Science, University of Elimam Elmahdi \\ ${ }^{2}$ Department of Information Systems, University of Bisha \\ Sudan
}

\begin{abstract}
In this study, we develop a reliable and highperformance multi-layer feed-forward artificial neural networks (MFANNs) model for predicting gender classification. The study used features for a set of 450 images randomly chosen from the FERET dataset. We extract the only high-merit candidate parameters form the FERET dataset. A discrete cosine transformation (DCT) is employed to facilitate an image description and conversion. To reach the final gender estimation model, authors examined three artificial neural classifiers and each extremely performs deep computation processes. In addition to the MFANNs, artificial neural networks (ANNs) classifiers include support vector regression with radial-basis function (SVR-RBF) and $k$-Nearest Neighbor (k-NN). A 10-folds cross-validation technique $(\mathrm{CV})$ is used to prove the integrity of the dataset inputs and enhance the calculation process of the model. In this model, the performance criteria for accuracy rate and mean squared error (MSE) are carried out. Results of the MFANNs models are compared with the ones that obtained by SVR-RBF and $k-N N$. It is shown that the MFANNs model performs better (i.e. lowest MSE $=0.0789$, and highest accuracy rate $=96.9 \%$ ) than SVR-based and $k-N N$ models. Linked the study findings with the results obtained in the literature review, we conclude that our method achieves a recommended calculation for gender prediction.
\end{abstract}

\section{Introduction}

Gender detection is an indispensable biological metric and plays a significant role in many human applications. These applications vary to represent immigration, border access, law enforcement, defence and intelligence, citizen identification, and banking [1]. A daily increasing demand for a reliable gender classifier motivates researchers for continuous competing in developing algorithms that solve gender prediction and fixing verification problems [2]. Nowadays, gender prediction represents a primary factor in all human-based techno-systems. A study [3] defines gender detection, as a convenient, verifiable, and inexpensive biometric feasible technique that widely used for human classification. Recently, a number of gender detection methods have been discovered. However, this field is still open and expecting nonexpensive and more accurate algorithms to be announced [4]. Head and face zones are the most important human parts that contain several valuable gender characteristics and each feature is mature enough to be examined to validate a gender class [5]. Based on morphological structure [5-6], the primary difference between male and female can be mentioned in many points. In summary, a study in [6] determined these elementary points as: face size and dimorphism, skull appearance at the forehead region, the cheekbones, the superior rim in the eye orbital area, and the chin. Figure 1 below depicts the skull variations between male and female. Considering human classification, indeed other indications and feelings such as mood, identity, and ethnicity are very prior in many gender prediction and classification techniques [7].

In general, the input features for the prediction system are divided into two main categories; named local properties and global ones. The global features involve geometric dimensions and occlusion. However, the local category covers the batches which are very necessary for computing vector [8]. Before computations, the input features are preprocessed and organized. This step requires a proper measuring and orchestrating the merit parameters that ultimately enhance the classifier power. The study used the FERET dataset to grant the quality of the input records and strengthening the outputs computations.

The previous studies proved that researchers have reached consensus on the accuracy of the FERET dataset usability [7-9]. Authors are very grateful for the National Institute of Standards and Technology (NIST) for permitting us to use the FERET dataset [see Appendix]. From the RERET dataset, a set of 450 images data points (records) are randomly selected. Each data point is an array of six inputs fields and a single output feature. Each field represents one or more input variables in the inputs set. In our ANNs prediction models, the input matrix contains variables for flags, kind, name, date, extension, and modifiers which are determined to 
represent the exact image metrics. These metrics are abstracted from the image size, type, name, glasses, eyes, hair, position, etc. All images extensions are of type portable network graphics (.png). In this study the only output parameter is gender. A set of the selected features have been encoded to form a single and standard numerical array for the images. To facilitate the computation complexity, the system normalized the encoded numerical values. It is a robust transformation method and very suitable for image processing and objects recognition. DCT is the popular image descriptors and transformation techniques used in training and testing phases to support the ANNs' classifiers that perfectly compute the outputs. It extracts the maximum merits features from the original image, forms the image matrix, and simply forwards them as inputs to the input layer for gender computation model [5]. The golden goal of this paper is to build, with the help of ANNs prediction tools and use the FERET dataset, a reliable model for gender prediction characterizes with low errors and cost too. Indeed, many proposals for gender prediction have been posted, but very often, ANNs' techniques are the fittest candidates that perfectly performed gender examination [10], [11].

The study uses three powerful neural intelligent mechanisms (MFANNs, SVR-RBF, and $\mathrm{kNN}$ ) with a deep model derivation and successfully constructed an accurate gender prediction model. These ANNs' techniques characterized by a high ranking in building models for prediction, classification, and promote the calculation processes. The study evaluates the classifiers' performance measured by computing the accuracy rate and MSE. The results proved that our model for gender prediction is highly recommended and the MFFANNs registered the highest rates ( $\mathrm{R} \& \mathrm{SEE}$ ). In summary, our neural networks classifiers' performance criteria can be ranking as MFFANN, SVR-RBF, and KNN. The rest of the study is organized as follows: Section 2 shows the previous related works. Section 3 gives the method used and overviews ANNs' classifiers techniques. Section 4 outlines the system framework and the dataset used for gender detection protocol. While Section 5 represents the results and discussion. Finally, Section 6 concludes the study and followed by references.

\section{Related work}

In recent times, growing demands for receiving a reliable gender prediction technique is extremely recorded and open this field for a deep research works. This fact encourages researchers for a continuous developing to reach the best tool for gender prediction. Indeed, many proposals in gender verification have been published $[1,2,4,12,13,14$, and 15]. Table 1 below summarizes the significant articles accomplished in this field with their findings. As a result of these researches, it can be concluded that the use of ANNs is highly recommended and is a promising approach to be used in gender classification applications. Particularly, obtaining ANNs' prediction and classification algorithm is very feasible and accurate. Articles in [3, 10, and 14] share the basic ideas for gender detection and face recognition. Using a mobile application, a study in [11] introduced a conventional ANNs base for gender detection. Instead of FERET dataset, the study used a private video dataset. The study outputs were pretty promising in adequate lighting conditions; however, it was failed to validate face and gender during moonlight conditions. In [16], the study examined a hieratical approach for a multiview facial recognition to reach the target gender. To serve voting application schemes, the study multiplied images from different viewpoints and created a valuable dataset and enhanced the evaluation outputs. Paper [17], is more nominated to use in verification for how fast gender recognition performs. To obtain the results, three different face processing levels were set. These levels include: a superordinate face categorization level; a face familiarity level; and verifying a face from a target person level. The study used 27 subjects to test and validate the results. This methodology elapses the system only 0.25 seconds to figure out the targeted person's gender from the crowd.

A study [10] employed multi agent tool for classify people extracting only age and gender from the image. It was carried out under an uncontrolled condition, particularly brightness, contrast, and saturation. Great efforts were hired to refine the image quality and integrated techniques used. The system performs classification in real world very well. Based on face attributes, [18] predicts gender and age by analyzing the dataset of four factors. In the analysis, the study includes factors for age, neural network depth, penetrating, and strategy. The study reached a recommended finding for gender recognition and age estimation. In order to boost up gender recognition criteria, papers $[14,19]$ combined both the face inner cues and outer cues with neural technique. The study results claim that the external cues quietly improve prediction performance for gender recognition pattern. Furthermore, the logic inference system improves the prediction results. When SVR-based used, the results show that unconstrained database performs better results than that of the constrained database with the averages obtained $93.35 \%$ and $91.25 \%$, respectively. Paper [14], a gender recognition performed by using: 1) neural faces; 2) expressive faces, and 3) occluded faces.

To obtain the results, [14] compared global/local applications/Grey level/PCA/LBP features and three classifiers. Also, three statistical test across two 
performance measures were employed to support the conclusion of local models surpass the global ones with different types of training and testing faces datasets. However, global and local models performed equal outputs when running the same training and testing faces data points. Using human giants in the image sequence, the study [23] investigated gender classification. A study [23] exploited canonical correlation analysis and minimize the errors across a large dataset.
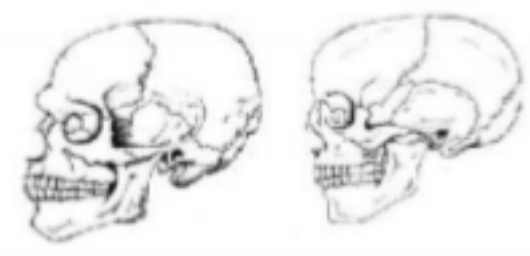

Figure 1. Skull gender variations between Male (left) and female (right)

\section{Method and classifiers overview}

\subsection{Method}

In this model, models for gender prediction using ANNs' intelligent classifiers are used. To achieve the best results, models for MFANNs, SVR-RBF, and $\mathrm{kNN}$ were constructed and operated. Figure 2 gives a schematic diagram for our ANNs' classifiers architecture and the following points outline the method:

1. From the source FERET dataset, a set of 450 data point is cropped.

2. DCT is a dynamic and flexible image descriptor technique used to transform images in the training and testing phases.

3. MATLAB R2010a is used to perform ANNs computation.

4. Input features for the elite patches and the candidate are measured and extracted for the cropped data points, preprocessed, and forwarded to the input layer.

5. ANNs' classifiers (i.e. MFANNs, SVR-RBF, and $\mathrm{k}-\mathrm{NN}$ ) models are built to calculate the performance metrics.

6. The linear output function predicts the class type as male or female.

To perfectly compute the outputs, a 10 -fold CV is used [20] and the averages reported. During the training and testing phases, the male and female classes are coded as 1 and 0 respectively.

\subsection{MFANNs}

MFANNs is a powerful subset of machine learning technique in which multilayered feed-forward learn from the vast amount of data. It is an intelligent way in neural computing used for evaluating prediction and classification performance. A study [21], well introduced and presented the MFANNs. It imitates the human brain neurons behavior for data processing. MFANNs orchestrates a single input layer, two or more hidden layers, and a linear single output layer. Initially, the training dataset is nurtured to the system via the input layer and each neuron propagates its computed outputs and forwarded it to the next corresponding neurons across a system of coherent interconnected layers.

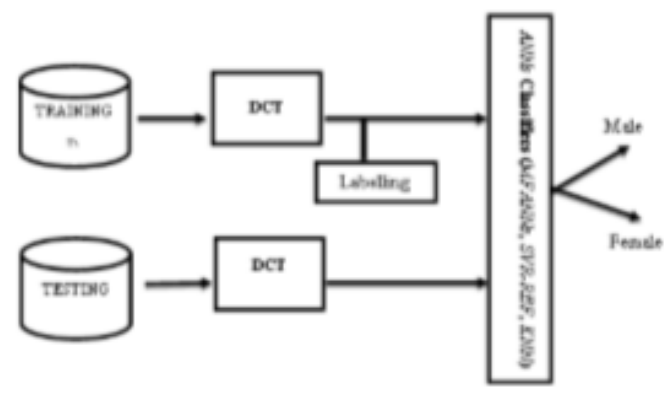

Figure 2. A Schematic ANNs classifiers

This process adapts the MFANNs errors and the final output prediction calculated and presented in the output layer. Equation (1) used to calculate the mean squared error and Figure 3 gives a typical MFANNs architecture. Where Ui are inputs, hi(.) and $\mathrm{Xi}($.$) are the first and the second hidden layers'$ computations, respectively, and y is the output class. The back-propagation algorithm is the ultimate method to perform the finest result [11].

$$
E(t)=\frac{1}{2} \sum\left(y_{j}(t)-d_{j}(t)\right)^{2}
$$

where $\mathrm{E}(\mathrm{t})$ is MSE at any time $\mathrm{t}$; $\mathrm{yj}(\mathrm{t})$ is the predicted output; $\operatorname{dj}(t)$ is the desired output.

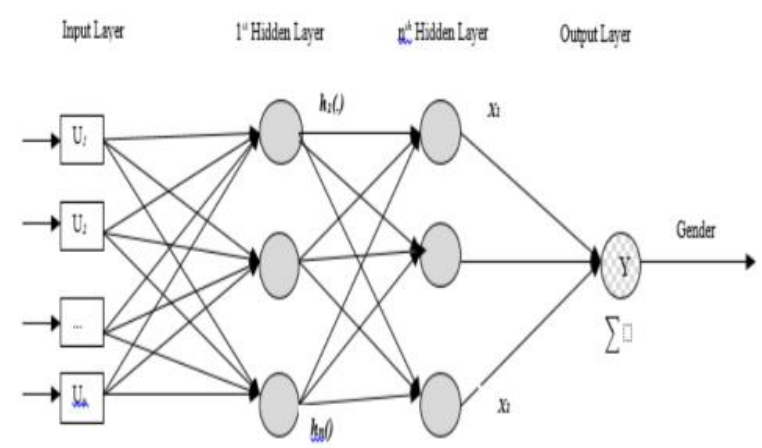


Figure 3. A typical MFANNs

\subsection{SVR-RBF}

SVR-based, is a recommended ANNs prediction algorithm commonly used in gender detection applications. SVR-RBF is a super non-linear form prediction method can be used to reach an optimal gender recognition result. The following equations (2-5) are derived to calculate the SVR-based classifier [22], [24]:

$$
\begin{aligned}
& f(x)=\langle\omega, \phi(x)\rangle+b ; \quad \omega, x \in \mathfrak{R}^{d} ; \mathrm{b} \in \mathfrak{R} \\
& \text { where } \\
& \bar{\omega}=\sum_{i=1}^{f}\left(\alpha_{i}-\alpha_{i}\right) \phi\left(x_{i}\right), \\
& \langle\omega, \bar{\phi}(x)\rangle=\sum_{i=1}^{f}\left(\alpha_{i}-\alpha_{i}{ }^{*}\right)\left\langle\phi\left(x_{i}\right), \phi(x)\right\rangle=\sum_{i=1}^{l}\left(\alpha_{i}-\alpha_{i}\right) K\left(x_{i}, x\right), \\
& \bar{b}=\frac{1}{2} \sum_{i=1}^{b}\left(\alpha_{i}-\alpha_{i}^{*}\right)\left(K\left(x_{i}, x_{r}\right)+K\left(x_{i}, x_{s}\right)\right)
\end{aligned}
$$

where $\phi$ is nonlinear mapping of the input space onto a higher dimension feature space; $x r$ and $x s$ are support vectors. Eq. (3) can be written as follows if the term $b$ is accommodated within the kernel function:

the term $\mathrm{b}$ is accommodated within the kernel function:

$$
\sum_{i=1}^{l}\left(\alpha_{i}-\alpha_{i}^{*}\right) \mathrm{K}\left(\mathrm{x}_{\mathrm{i}}, \mathrm{x}\right)
$$

The final derived equation for SVR-RBF is:

$$
K\left(x, x^{\prime}\right)=\exp \left(-\frac{\left\|x-x^{\prime}\right\|^{2}}{2 \rho^{2}}\right)
$$

Where $\rho$ is the width of the RBF kernel.

$\mathrm{K}$-nearest neighbors ( $\mathrm{k}-\mathrm{NN})$, is a simple nonparametric machine learning technique widely used to classify data-based similarities. K-means developed to perform anomaly detection analysis when reliable parametric estimates of probability densities are unidentified. It uses the Euclidean distance metrics to categorize the new point (testing) into the existing groups (training).

\section{Dataset generation}

In this study, the primary FERET database is used because authors were get a permission and offered an access right to use it for a research work (NIST, 2017). The amount of the FERET dataset consists of 14051 data points' 8-bit grayscale images of human heads with views ranging from frontal to left and right profiles. The characteristics and the descriptive statistics for the dataset was well introduced in [7-9] and it is access right distributed by The National Institute of Standards and Technology (NIST). From the FERET dataset, the study used only a subset of 450 (male is 237, female equals 213) data records. Images named in an integer sequence of the form (nnnnnxxfffq_yymmdd.ext). This long string file name organized as: the first 5 (nnnnn) digits represent a file name, the next two digits indicate the kind of imagery (i.e. fa: for the frontal expression, and fb for alternative facial expression), three fff binary digits represent a single flag (a: if the image is releasable for publication; b:if image is histogram adjusted; and c: if image was captured), a single bit named $q$ for a modifier such as(i.e. if $\mathrm{q}=\mathrm{a}$ : glasses worn; if $\mathrm{q}=\mathrm{b}$ : duplicate with different hair length; if $\mathrm{q}=\mathrm{c}$ : glasses worn and different hair length; if $\mathrm{q}=\mathrm{d}$ : resized and adjusted; if $\mathrm{q}=\mathrm{e}$ : clothing has been retouched; if $\mathrm{q}=\mathrm{f}$ and $\mathrm{g}$ : for image brightness reduced by $40 \%, \quad 80 \%$, respectively; if $\mathrm{q}=\mathrm{h}, \mathrm{i}$, and $\mathrm{j}$ : for image sized has been reduced by $10,20 \%, 30 \%$, respectively, and the three fields (yymmdd) represent the date in year, month, and day format. The file extension defines the data type inside a file (.png).

Therefore, the study consists of: fb equal 400 subjects, fa is 50 subjects, images with $q=a$ is 35 , and so on. DCT converts a picture from its original domain to the frequency domain and it is used for the real numbers only. Based on image frequencies, DCT divides images into different parts. In quantization phase, the minor frequencies neglected and the only main frequencies are extracted in the prediction phase [5]. The DCT is a robust gender prediction system through the equations Eq. (6) and Eq. (7).

$$
\begin{aligned}
& D(i, j)=\frac{1}{\sqrt{2 N}} C(i) C(j) \sum \sum_{s=0}^{N=0} \sum_{j=0}^{N-1} p(x, y) \cos \left[\frac{(2 x+1) i \pi}{2 N}\right] \cos \left[\frac{(2 y+1) j \pi}{2 N}\right] \\
& C(u)=\left\{\begin{array}{llll}
\frac{1}{\sqrt{2}} & ; & \text { if } & u=0 \\
1 & ; & \text { if } & u>1
\end{array}\right\}
\end{aligned}
$$

where $\mathrm{m}(\mathrm{x}, \mathrm{y})$ represent the $(\mathrm{x}, \mathrm{y})$ th element of the image in matrix $p$, and $i$ and $j$ are coordinates in the transformed image. $\mathrm{N}$ is the size of the block that the 
DCT perform. Eq. (6) computes one entry $(i, j$ th) of the transformed image from the pixel values of the original matrix [5].

\section{Results and discussion}

The elementary process for the system can be summarized in Figure 4 which illustrates the image selection steps. First, the original image is randomly cropped from FERET dataset as in Fig. 4(a) randomly choose an image. Second, resized the selected image as in Fig. 4(b). Third, convert the target image into an RGB form (red, green, and blue) as in Fig.4(c). Eq. (8) used to outputs any given RGB color vector of the values $\mathrm{a}, \mathrm{b}$, and $\mathrm{c}$, respectively [16]. For the preprocessing process a normalization is an indispensable way that makes a descriptor independent from lighting changes.

$$
\text { length }=\sqrt{a^{2}+b^{2}+c^{2}}
$$
255.

where $a, b$, and c are any color values from 0 to

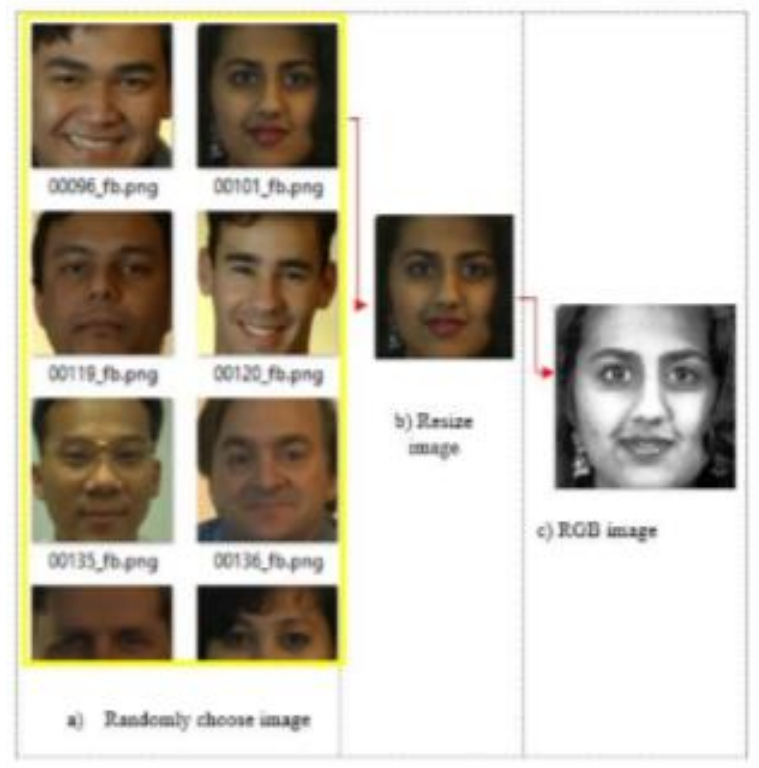

Figure 4. Illustration of image selection

Figure 5 gives the performance measures for the ANNs gender prediction outputs. In this figure, the arrow gives the direction of gradient while the arrow's length shows the magnitude, and the direction of arrows indicates the direction of change in intensity. The angles range from 0 to 180 degrees because the study employs unsigned gradients paradigm. An unsigned number properly represents a negative and positive number and gives high performance in gender determination than the other algorithms. The outcome number is chosen based on both the direction with the corresponding number for the magnitude.

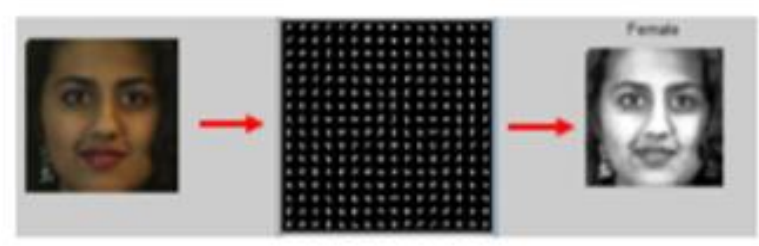

Figure 5. Performance measures for ANNs Output.

ANNs procedural process model is illustrated in Figure 6 below. It summarizes the procedure model steps starting from network initialization until gender prediction.

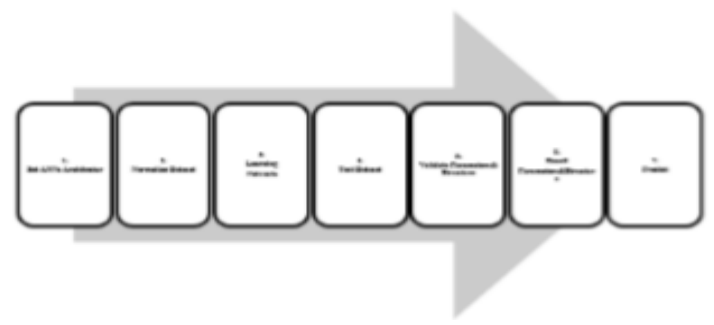

Figure 6. ANNs Procedure Steps

In order to achieve high output rates, the dataset is precisely divided into $80 \%$ and $20 \%$ for the training dataset, and the testing dataset, respectively. The training phase represents an initial part for the prediction system construction. However, many public training algorithms can be used for adapting the network and the Levenberg-Marqurdit is a recommended one. The testing phase examines the performance measures of the classifier and validates the system accuracy. The performance metrics of ANNs techniques are calculated by using 10-folds $\mathrm{CV}$ and reports the arithmetic averages for the accuracy rate and MSE. The following Eq. (9) and Eq.(10) used to calculate the R and the standard error of estimate (SEE) metrics.

$$
\begin{gathered}
S E E=\sqrt{\frac{1}{n} \sum_{i=1}^{n}\left(Y-Y^{\prime}\right)^{2}} \\
R=\sqrt{1-\frac{\sum_{i=1}^{n}\left(Y-Y^{\prime}\right)^{2}}{\sum_{i=1}^{n}(Y-\bar{Y})^{2}}}
\end{gathered}
$$


where $\mathrm{n}$ is the number of data points used for testing, $\mathrm{Y}$ is the measured value, $\mathrm{Y}^{\prime}$ is the predicted value, $\mathrm{Y}$ is the average of the measured values, $\mathrm{Y}^{\prime}$ is the average of the predicted values.

Figure 7 gives our neural networks prediction performance metrics.

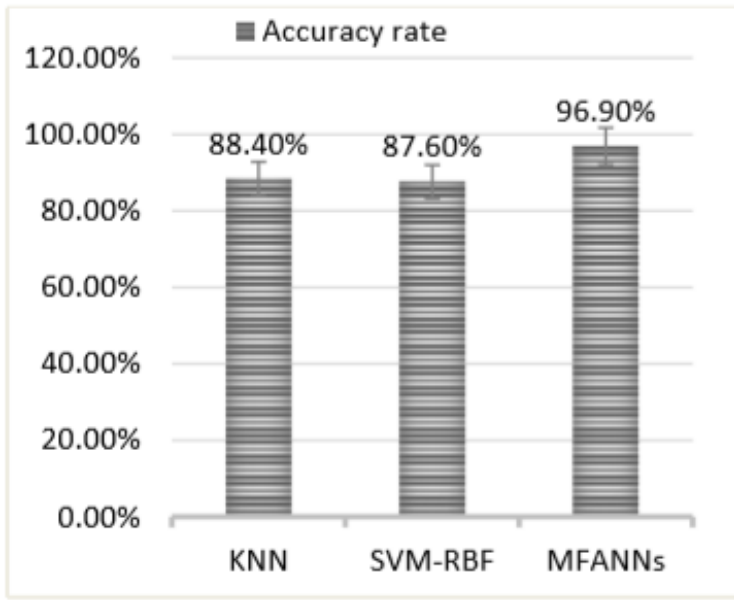

Figure 7. ANNs accuracy rate and errors

Table 1. Summary of the ANNs related works

\begin{tabular}{|c|c|c|}
\hline Auchors, Year & Clessifiers & Accuracy Rate \\
\hline Our study & $\begin{array}{c}\text { MFANNS, } \\
\text { SVR-RBF, } \\
\text { KNN }\end{array}$ & $96.9 \%$ \\
\hline Andreu Yasmina, et al., (2014) & $s n M$ & $93.35 \%$ \\
\hline Palmer Matthew A, et al., (2013) & $\begin{array}{c}\text { Estimation } \\
\text { (Error) }\end{array}$ & $95.0 \% 6^{*}$ \\
\hline Shan Caifeng, et al., (2008) & $s h M$ & $97.2 \%$ \\
\hline Mansanet Jordi, et al., (2016) & Local-DNN 9 & $96.25 \%$ \\
\hline Wong and Amini, (2016) & $\begin{array}{c}\text { Decision } \\
\text { Tree }\end{array}$ & $75.8 \%$ \\
\hline Baluja and Rowley, (2007) & SWM & $93.0 \%$ \\
\hline
\end{tabular}

It is recommended that MFANNs performs better results than SVR-RBF and k-NN. Compared our findings versus the ones shown in Table 1, authors claim that this method performs the highest accuracy results (i.e. accuracy rate is $96.9 \%$ and SEE limit to
0) using multi-layer feed-forward ANNs architecture. This study proved that MFANNs findings are even better than SVRRBF. Also, the study concludes that $\mathrm{k}-\mathrm{NN}$ algorithm is not a recommended way in predicting gender detection applications.

Table 2 shows the MFANNs structure that gives the best results for gender detection evaluation. Promoting the MFANNs technique requires a deep organizing for its neural elements layering architecture, which coordinates one input layer for inputs, two hidden layers each supported by several neurons and a tansigmoid activation function, and a linear-based activation function for the output predictions. This system configurations reached after a long examination for all network parameters individually and observed each parameter's contributions on the outputs.

Table 2. ANNs Performance Metrics for Gender Prediction

\begin{tabular}{|c|c|c|c|}
\hline $\begin{array}{c}\text { Gender } \\
\text { Prediction }\end{array}$ & Classifier & $R$ & $S E E$ \\
\hline \multirow{3}{*}{$A N N s$} & $k-N N$ & $88.4 \%$ & 0.0883 \\
\hline & $S V M-R B F$ & $87.6 \%$ & 0.0931 \\
\hline & $M F A N N s$ & $96.9 \%$ & 0.0789 \\
\hline
\end{tabular}

Figure 8 describes the MFANNs validation measures, which include: the learning rate (chosen as 0.02), a momentum (chosen as 0.5) and the best validation parameters are (selected as 0.078921 ) at epoch 6 for a single image which demonstrates the accuracy with MSE limits to zero.

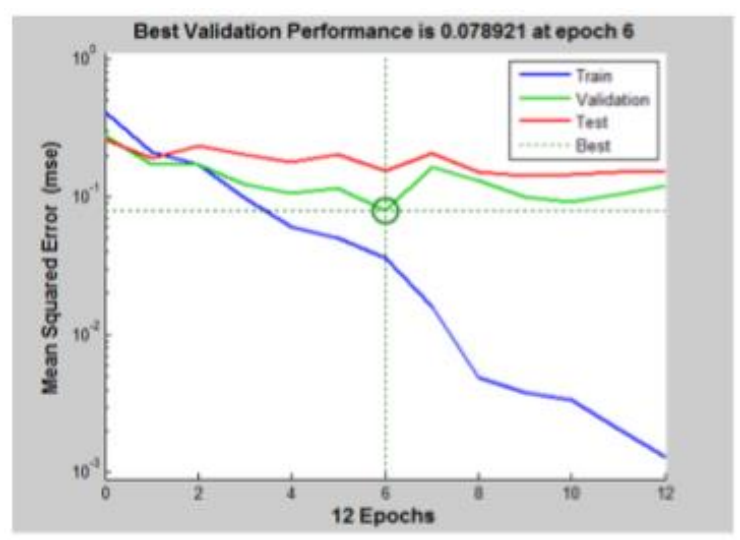

Figure 8. A MFANNs validation metrics.

\section{Conclusion}

The lack of a reliable and the best gender prediction system motivates researchers for a continuous developing in prediction algorithms, especially in the areas for a cosmetic surgery and the 
security applications. In summary, three robust machine learning techniques named MFANNs, SVRRBF, and k-NN models are used to predict the gender detection. A set of 450 subjects is selected from the FERET dataset and used for gender prediction system. Improving results computation, a 10 -folds CV technique is used and the performance averages for the accuracy rate and SEE values are reported. Results of our three-gender prediction can be listed in an ascending form as: MFANNs, SVR$\mathrm{RBF}$, and k-NN. It is shown that MFANNs registered the highest performance accuracy rate and lowest errors. Comparing the results achieved in this study versus the ones obtained in the previous related works, authors claim that the findings is a highly recommended and extreme-reliable for gender prediction. Future research can be extended to amplify the input features from the face area, iris, and eye detection to perform gender prediction.

\section{References}

[1] Baluja S. and Rowley H.A., (2007), "Boosting Sex Identification Performance", Int. J. Comput. Vision, Vol. 71, pp. 111-119.

[2] Fares A. E., (2016), "Real-Time Gender Classification by Face", International Journal of Advanced Computer Science and Applications, (IJACSA), Vol. 7, No. 3, pp.332-336.

[3] Ali Kh. S., Muhammad N. and Naveed R., (2013), "Optimized Features Selection for Gender Classification Using Optimization Algorithms", Turkish Journal of Electrical Engineering and Computer Sciences, pp.1479 - 1494.

[4] Eidinger E., Enbar R. and Hassner T., (2014), "Age and Gender Estimation of Unfiltered Faces", IEEE Trans. Inf. Forensics Secur., Vol. 9, pp.21702179.

[5] Shekar B.H. and Pilar B., (2015), "Discrete Cosine Transformation and Height Functions Based Shape Representation and Classification", Procedia Computer Science, Vol. 58, pp.714- 722.

[6] Graf A.B.A. and Wichmann, F.A., (2002), "Gender Classification of Human Faces", International Workshop on Biologically, Motivated Computer Vision, pp. 491-500.

[7] http://www.itl.nist.gov/iad/humanid/feret/feret_ master.html, (22th July, 2017).

[8] Phillips P.J., Wechsler H., Huang J., Rauss P., (1998), "The FERET Database and Evaluation Procedure for Face Recognition Algorithms", Image and Vision Computing J., Vol. 16(5), pp. 295-306.
[9] Phillips P.J., Moon H., Rizvi S.A., Rauss P., (2000), "The FERET Evaluation Methodology for Face Recognition Algorithms", IEEE Trans. Pattern Analysis and Machine Intelligence, Vol. 22, pp.1090-1104.

[10] González-Briones A., et al., (2018) “A multiagent system for the classification of gender and age from images", Computer Vision\& Image Understanding,

https://doi.org/10.1016/j.cviu.2018.01.012

[11] McCurrie M., et al., (2018) "Convolutional Neural Networks for Subjective Face Attributes", Image and Vision Computing, Vol. 78, October 2018, pp. 14-25.

[12] Xu Z., Lu L., and Shi P., (2008), “A Hybrid Approach to Gender Classification from Face Images", In Proceedings of IEEE International Conference on Pattern Recognition, pp.1-4.

[13] Wang X., Yang M., Shen L., (2016), "Structured Regularized Robust Coding for Face Recognition”, Neurocomputing, Vol. 216, pp.18-27.

[14] Andreu Y., and et al., (2014), "Face Gender Classification: A Statistical Study When Neutral and Distorted Faces are Combined for Training and Testing Purposes", Image and Vision Computing, Vol. 32(1), pp.27-36.

[15] Khashei M, Hamadani A Z, Bijari B., (2012), A novel hybrid classification model of artificial neural networks and multiple linear regression models, Expert Systems with Applications, Vol. 39, pp.26062620.

[16] Kim D., and et al., (2017), "MultiView Face Recognition from Single RGBD Models of the Faces", Computer Vision and Image Understanding, In Press, Accepted Manuscript.

[17] Chaudhry S. and Chandra R., (2017), "Face Detection and Recognition in an Unconstrained Environment for Mobile Visual Assistive System", Applied Soft Computing, Vol. 53, pp. 168-180.

[18] Antipov G., et al., (2017) "Effective Training of Convolutional Neural Networks for FaceBased Gender and Age Prediction", Pattern Recognition, Vol. 72, December 2017, pp. 1526.

[19] Alpaydin E., (2010), Introduction to Machine Learning, 2nd Ed., MIT press, London.

[20] Witten I.H., and Frank E., (2005), Data Mining: Practical Machine Learning Tools and Techniques, Morgan Kaufmann. 
[21] Schölkopf, B., Smola, A. J (2002) Learning with kernels: support vector machines, regularization, optimization, and beyond. Cambridge, MA: MIT Press.

[22] Mansanet J., Albiol A., and Paredes R., (2016) "Local deep neural networks for gender recognition", Pattern Recognition Letters, Vol. 70, pp. 80-86. [23] Shan C., Gong S., and McOwan W. P., (2008) "Fusing gait and face cues for human gender recognition", Neurocomputing, Vol. 71(10-12), pp.1931-1938.

[24] Cristianini N. and Shawe-Taylor J., (2000) "An introduction to support vector machines and other kernel-based learning methods", Cambridge, UK: Cambridge University Press.

\section{Acknowledgements}

We would like to thank the National Institute of Standards and Technology (NIST) for permitting us to use the FERET dataset "Portions of the research in this paper use the FERET database of facial images collected under the FERET program, sponsored by the DOD Counterdrug Technology Development Program Office". 
Appendix A

\section{Color FERET Database Release Agreement}

To advance the state-of-the-art in face recognition, the FERET database will be made available to researchers in face recognition on a case-by-case basis only. All requests for the FERET to researchers in face recognition on a case-by-case basis only. All requests for the FERET
database must be submitted vin the NIST website. To receive a copy of the database, the database must be submuitted via the NIST website. To receive a copy of the database, the
researcher must sign this document and thereby agree to observe the restrictions listed herein Failure to observe the restrictions in this document will result in access being denied for the
balance of the FERET database and being subject to civil damages in the case of publication of images that have not been approved for release, a violation of restriction 3 below. The database will be available to researchers via NIST website download only. The images are available ppm format, no other image formats or methods of distribution will
researcher(s) agrees to the following restrictions on the database: 1. The database will not be further distrnbuted, published, copied, or further disseminated in any
way or form whatsoever, whether for profit or not. This includes further distributing, copying or disseminating to a facility or organization unit in the requesting university, organization, of company.

2. The images will only appear in technical reports, technical papers, and technical documents reporting on face recognition research. There will be no more than 15 images used at a time in a publication.

3. All documents and papers that report on research that uses the FERET database will acknowledge the use of the FERET database. Use of the FERET database will be acknowledged as follows: "Portions of the reseasch in this paper use the FERET database of facial image collected under the FERET program, sponsored by the DOD Counterdrug Technologg

a. P.J. Phil1ips, B. Wechslex, J. Huang, D. Rauss, algor whe Frigr database and evaluation procedure for face recognition

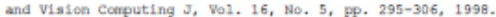

b. P.J. Phillips, B. Moon, S.A. RLzv1, P.J. Rauss,

"The FERST Evaluation Nethodology for Face Recognition A1gorithes,"

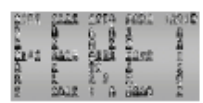

\section{Appendix B}

a Color FERET database download account has been created for you Mhmed Yosuf UoI

Color FERET Database Account Administrator <colorferet@Mtst. bopas 7. to me

Thank you for your request to download the Color FERET image database. This is a automated reply.

You have been given an account with:

\begin{tabular}{ll} 
usemame & XXXXXXX \\
password & $X X X X X X X$ \\
\hline
\end{tabular}

They usemame and password are needed to allow you to complete HTTP

authentication with our webserver. Do not share the account username

and password with anyone else.

The Color FERET database is in a "tar" archive of approximately 8.5 gigabytes. It also comes with an "md5" file containing the "tar" archives's checksum to verify the download succeeded.

-W-r-r-r- 1 root root 8579819520 Feb $1618-28$ colorferet.tar

49 Feb $2501: 28$ colorferet.tar.md5

If your computer has a command-line program called "wget", you can download
both fles into a directory "colorferet" (which will be created if it doesn't

already exist) by simply executing a single command:
wget $-v-c-r \rightarrow$-no-parent - -no-host-directories $-e$

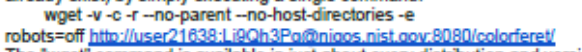

of Linux. If it wasn't installed by defaut it

kage repository

For Apple's Mac OS X operating system, a similar downloader command-line

program "curl" is present by default

And if your computer doesn't have a URL downloader "wget" installed or available, there are several other options for downloading. Please see

hittos:/wwww nistaovititliadlimage-arounicolor-feret-database

It might be easier to download the 2 files individually: wget -v - c htto://user21638:Li8Qh3Pg@nigos.nist.gov:8080/colorfereticolorferet.tar

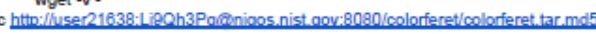

If for some reason, those commands fail, there are alternative commands rou can try 17) that breaks HTTP a bug in 'wget version 1.13 .4 (confirmed

run:

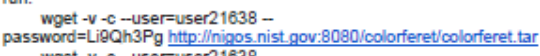

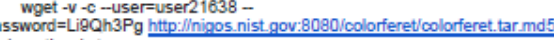
and another is to run:

challenge $h$ htp:I//user21638:Li9Ch3P g@inigos nist.gov:8080/colorfereticolorferet.tar

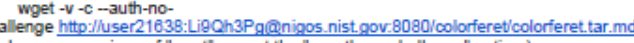

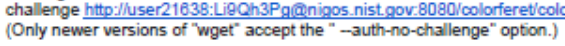

And another option is to instead use the "curl" program:

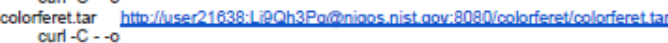

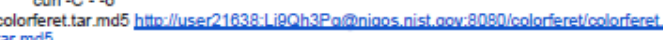
$\underline{\text { tar.mot5 }}$

Make sure that you download the files to a directory on a flesystem that supports files as large as 8.5 gigabytes. One flesystem that DOES NOT is
Windows' FAT flesystem - all files on it must be less than 2 gigabytes.

If the "wget" command fails to download the 2 files (for example, If the network connection is broken for some reason), the " $-c$ " option assures the

Once you're done downloading, if your computer has the command-line program "md5sum", you should verify the "tar" archive's checksum by

md5sum -check < colorferet.tar.md5 Then you can safely extract the database files from the pieces of the

$$
\operatorname{tar}-\mathrm{x}-\mathrm{f} \text { colorferet.tar }
$$

If you're unable to download the database, we can only advise that you seek help from computer-savvey people on your end. NIST has neither the
resources nor the capability to debug failed downloads.

The account will be deleted automatically in a few weeks. The exact amount of time will depend on several factors, including the webserver load and

Thanks,

- The NIST Color FERET Team

손

Rasheed Zayid <rasheedzayid16@gmail.com> Aug 22, 2017. to eazayid 